\title{
ON THE GENERATION OF THE HUBBLE SEQUENCE THROUGH AN INTERNAL SECULAR DYNAMICAL PROCESS
}

\author{
Xiaolei Zhang ${ }^{1}$ \\ ${ }^{1}$ US Naval Rsearch Laboratory, 4555 Overlook Ave. SW, Washington, DC 20375, USA
}

\begin{abstract}
The secular evolution process, which slowly transforms the morphology of a galaxy over its lifetime, could naturally account for observed properties of the great majority of physical galaxies if both stellar and gaseous accretion processes are taken into account. As an emerging paradigm for galaxy evolution, its dynamical foundation had been established in the past few years, and its observational consequences are yet to be fully explored. The secular evolution picture provides a coherent framework for understanding the extraordinary regularity and the systematic variation of galaxy properties along the Hubble sequence.
\end{abstract}

Keywords: Generation of the Hubble Sequence, Secular evolution of galaxies

\section{Introduction}

The dominant view over the past few decades has been that the structural properties of galaxies remain largely unchanged unless galaxies were perturbed by violent events such as mergers (Toomre \& Toomre 1972). This view is particularly favored by the currently popular hierarchical clustering/cold dark matter (CDM) paradigm of structure formation and evolution (Peebles 1993 and the references therein). In the early 1980s, photometric and kinematic evidence in the buldges of late-type galaxies, and hints from N-body simulations of barred galaxies which incorporated a dissipative gas component, prompted several investigators to speculate that a fraction of late-type bulges might be formed from gas accretion under the bar potential (Kormendy 1982; Combes $\&$ Sander 1981). These early observations and speculations have since been further developed into one version of the secular evolution scenario, which emphasizes the role of dissipative gas accretion in the formation of the socalled "pseudo bulges" in late type galaxies (Kormendy \& Kennicutt 2004 and the references therein).

Even though the role of mergers during the early phases of galaxy assembly is still to be assessed, growing evidence has shown that at least since $\mathrm{z} \sim 1$ 
the rate of merger appears to have been significantly reduced (Conselice et al. 2003), and during subsequent time the significance of merger in transforming galaxy morphology is likely to be overshadowed by the slower secular evolution process (Kormendy \& Kennicutt 2004).

However, secular evolution involving gas alone under barred potential leads to some apparent paradoxes. First of all, as emphasized by Andredakis and coworkers (Andredakis, Peletier, \& Balcells 1995), the continuity of the galaxy properties across the entire Hubble sequence, highlighted for example by the continued variation of Sersic index $\mathrm{n}$ in fitting the bulge surface density profile, indicates that there is not an apparent break in the formation mechanism between the late-type disk galaxies and mid-to-early-type disk galaxies. However, due to the paucity of gas compared to stars in most galaxies, manifesting as a gaseous-to-stellar mass ratio of $1 / 10$ or less (which is true even for high-redshift late-type disk galaxies, presumably a result of rapid star formation following the accretion shocks of dissipative disk formation, D. Sanders 2002 private communication; F. Combes 2004 private communication), there simply is not enough of a reservoir of gas for use to build up the bulges of intermediate Hubble type galaxies such as our own, which has a bulge mass comparable to the disk mass, not to say for galaxies of even earlier Hubble types. Secondly, bulges of intermediate to early type galaxies, including our own, consists mostly of stars of very old age (Jablonka, Gorgas \& Goudfrooij 2002), and could not have been built up by the secular accretion of gas over a Hubble time which subsequently formed stars. Even though Kormendy and Kennicutt (2004) argued that some of these bulges may be termed "classical", i.e., they could have formed out of the dissipative collapse at an early stage of galaxy formation, they nonetheless pointed out that a good fraction of these apparently old bulges have stellar kinematics which were rotation-dominated, and are related to the kinematics of their disks, hinting at a secular evolution origin for their formation.

These and other apparent paradoxes, such as the existence in some galaxies of dense core in the central region which appears to be formed by radial gas accretion and yet is kinematically decoupled from the bulge stars, can be naturally resolved if we allow also the possibility of stellar accretion. Such a possibility was not explored in the past decades mainly due to a theoretical barrier, i.e., the well-known result that the stellar motion in a galaxy containing quasi-stationary non-axisymmetric patterns conserves the Jacobi integral in the rotating frame of the pattern (Binney \& Tremaine 1987), and the orbital motion of stars under such potentials generally will not exhibit secular delay or increase, since there is no wave and disk star interaction except at the waveparticle resonances which are usually localized for quasi-steady wave patterns (Lynden-Bell \& Kalnajs 1972). 


\section{Physical Mechanisms for Producing the Secular Morphological Evolution of Galaxies}

It was first demonstrated in Zhang $(1996,1998,1999)$ that secular orbital changes of stars across the entire galaxy disk are in fact possible due to the collective instabilities induced by the unstable density wave modes such spirals, bars, as well as by the skewed 3D density distributions reflected in the twisted isophotes of many high-redshift galaxies. These skewed global patterns were shown to lead to a secular energy and angular momentum exchange process between the disk matter and the wave pattern, mediated by a local gravitational instability, or a collisionless shock, at the potential minimum of the pattern (Zhang 1996). The integral manifestation of this process is an azimuthal phase shift between the potential and density spirals, which results in a secular torque action between the wave pattern and the underlying disk matter. As a result of the torquing of the wave on the disk matter, the matter inside the corotation radius loses angular momentum to the wave secularly and sinks inward. The wave carries the angular momentum it receives from the inner disk matter to the outer disk and deposits it there, causing the matter in the outer disk to drift further out.

Closed form evolution rates can be derived for this process. In the quasi steady state, the rate of angular momentum exchange between a skewed density wave pattern (spiral, bar, etc.) and the basic state of the disk, per unit area, is given by (Zhang 1996, 1998)

$$
\frac{\overline{d L}}{d t}(r)=-\frac{1}{2 \pi} \int_{0}^{2 \pi} \Sigma_{1}(r, \phi) \frac{\partial \mathcal{V}_{1}(r, \phi)}{\partial \phi} d \phi,
$$

which, for two sinusoidal waveforms, is given by

$$
\overline{\frac{d L}{d t}}(r)=(m / 2) A_{\Sigma} A_{\mathcal{V}} \sin \left(m \phi_{0}\right)
$$

where $A_{\Sigma}$ and $A_{\mathcal{V}}$ are the amplitudes of the density and potential waves, respectively, and $\phi_{0}$ is the phase shift between these two waveforms.

The orbital decay rate it induced on a disk star (or a gas clump) can be derived from equation (2) as

$$
\frac{d r}{d t}=-\frac{1}{2} F^{2} v_{0} \tan (i) \sin \left(m \phi_{0}\right),
$$

where $F$ is the fractional wave amplitude (which is the geometric average of the fractional density and potential wave amplitude), $v_{0}$ is the circular velocity of the star, $i$ is the pitch angle of the spiral. $m$ is the number of spiral arms, respectively.

From the above expressions we can easily see why the skewness of the pattern is needed: It is the skewness of the mass distribution which leads to the 
potential/density phaseshift through the Poisson equation (Zhang 1996). Without phaseshift, there will be no secular angular momentum exchange between the wave and the disk matter at the quasi-steady state, and thus no secular mass redistribution. A perfect oval or triaxial mass distribution without any skewness will not be the direct driver for secular evolution.

For our own Galaxy, if we assume that over the past Hubble time the Galactic spiral pattern has an average $20 \%$ amplitude and 20 degree pitch angle, the orbital delay rate can be calculated using equation (3) to be about $2 \mathrm{kpc}$ of orbital decay per Hubble time, Therefore, a star in the Sun's orbit will not make it all the way in to the inner Galaxy in a Hubble time. However, the corresponding mass accretion rate across any Galactic radius inside corotation is about $6 \times 10^{9} M_{\odot}$ per Hubble time. A substantial fraction of the Galactic Bulge can thus be built up in a Hubble time.

Another important consequence of spiral-induced wave-basic state interaction is the secular heating of the disk stars. Since a spiral density wave can only gain energy and angular momentum in proportion to the pattern speed $\Omega_{p}$, and a disk star loses its orbital energy and angular momentum in proportion to its circular speed $\Omega$, an average star cannot lose the orbital energy entirely to the wave, the excess energy serves to heat the star. The resulting orbital velocity diffusion rate is given by

$$
D=\left(\Omega-\Omega_{p}\right) F^{2} v_{c}^{2} \tan (i) \sin \left(m \phi_{0}\right) .
$$

Using parameters appropriate for the Galaxy, this can quantitatively explain the observed age-velocity dispersion relation of the solar neighborhood stars (Zhang 1999; Wielen 1977). This secular heating process, since it originates from a local gravitational instability at the arms, serves to increase all three space velocities of stars. The vertical component of the velocity dispersion allows the stars to gradually drift out of the galactic plane as they spiral inward to become bulge stars. The corresponding energy injection into the interstellar medium, if used as the top-level energy input for turbulence cascade, can quantitatively account for the size-linewidth relation of the Galactic molecular clouds (Zhang et al. 2001; Zhang 2002).

The secular morphological evolution process leads to the Hubble type of an average galaxy to evolve from the late to the early (Zhang 1999). The secular evolution speed is expected to be faster for cluster galaxies than for isolated field galaxies, which were observed to evolve slowly, because the rate of secular evolution is proportional to the wave amplitude squared and pattern pitch angle squared (see, e.g., equations 3, 4), and cluster galaxies are found to have large amplitude and open spiral patterns excited through the tidal interactions with neighboring galaxies and with the cluster potential as a whole. This naturally accounts for the morphological Butcher-Oemler effect. 
The secular evolution of galaxies through the internal process mediated by skewed non-axisymmetric patterns is a rigorous consequence of the Newtonian dynamics and the assumption of global self-consistency. The analytical equations for the evolution rates (equations 3 and 4) are quantitatively confirmed in N-body simulations (Zhang 1998). Detailed comparison of the predictions of the secular evolution theory with the observed galaxy properties and with other theories of galaxy evolution can be found in (Zhang 2003). In what follows, we will highlight the role of secular evolution in generating the structural properties and scaling relations of galaxies.

\section{Generation of the Structural Properties of Galaxies Along the Hubble Sequence through Secular Evolution}

The properties of disk galaxies vary systematically along the Hubble sequence from the late to the early Hubble types. An important descriptor of the structural characteristics of disk galaxies is the rotation curve. After assembling hundreds of observed rotation curves for nearby disk galaxies, Persic, Salucci, \& Stel (1996) found that these rotation curves fall onto a twodimensional surface in the three-parameter space of normalized galactic radius, velocity, and the absolute magnitude. Since for the nearby galaxies the variation of the absolute magnitude corresponds to the variation of Hubble type (i.e., bigger and brighter galaxies generally have earlier Hubble types), this means that the rotation curve shapes also vary systematically for galaxies of varying Hubble types. In Figure 1, we plot three typical rotation curves generated using the data from Persic et al. (1996).

Using dynamical relations for equilibrium axisymmetric/spherically symmetric mass distributions, i.e. $V(R)^{2}=\frac{G M_{d y n}(R)}{R}$, as well as $M_{d y n}(R)=$ $\int_{0}^{R} \Sigma_{\text {proj }}(r) 2 \pi r d r=\int_{0}^{R} \rho(r) 4 \pi r^{2} d r$, where $M_{d y n}(R)$ is the dynamical or total mass within a galactic radius $\mathrm{R}$ (including the contributions from both the luminous and the dark components), and $\Sigma_{\text {proj }}(R)$ is the total projected surface density (disk plus spheroidal) at radius $R$, we can infer the underlying mass and surface density distributions corresponding to the different regimes for each type of rotation curves (c.f. Figure 1).

(I) Steeply rising solid body rotation curve, as observed for the inner regions of early type galaxies.

This regime is characterized by solid body rotation $\left(\Omega=\Omega_{0}=\right.$ constant, or a nearly linear rise of rotation velocity $\left.V(R)=\Omega_{0} R\right)$. Using $V(R)^{2}=\Omega_{0}^{2} R^{2}=$ $G M_{d y n} / R$, we obtain $M_{d y n}=\frac{\Omega_{0} R^{3}}{G}$, which implies a constant volume density $\rho=\rho_{0}=\frac{\Omega_{0}}{G} \frac{3}{4 \pi}$. Therefore the projected surface density can be obtained by equating $M_{d y n}(R)=\int_{0}^{R} \Sigma_{\text {proj }}(r) 2 \pi r d r$ and $M_{d y n}(R)=\frac{3}{4} \pi R^{3} \rho_{0}$, which gives $\Sigma_{\text {proj }}(R)=2 \rho_{0} R$, i.e. the solid-body regime corresponds to a constant column density $\rho_{0}$ and linearly rising projected surface density $\Sigma_{\text {proj }}(R) \propto R$. 


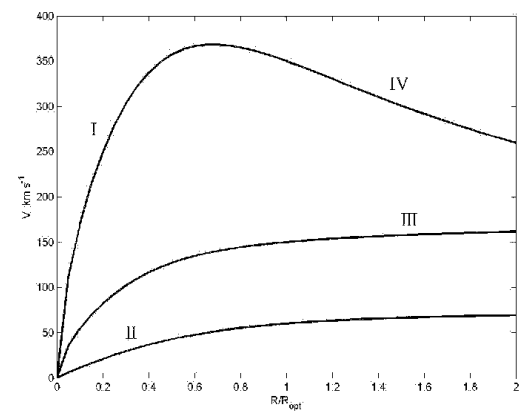

Figure 1. Typical rotation curves for disk galaxies of varying absolute magnitudes, as well as four characteristic regimes which are analyzed in the main text. Top: $M=18$; middle: $M=21$; bottom: $M=24$, where $M$ is the absolute magnitude of the representative galaxy (data from Persic et al [1996]).

(II) Gently rising rotation curve, as representative of the very late type disks, including most of the dwarf galaxies and low-surface-brightness (LSB) disk galaxies.

This regime can be shown to correspond to approximately constant projected surface density, as found to be the case for many LSB disks. Assume $\Sigma_{\text {proj }}(R)=\Sigma_{0}=$ constant, we obtain $M_{d y n}(R)=\int_{0}^{R} \Sigma_{0} 2 \pi r d r=\Sigma_{0} \pi R^{2}$, which we equate to $V(R)^{2} R / G$ to obtain $V(R)^{2}=\sqrt{\Sigma_{0} \pi R G}$, which indeed corresponds to a gently rising rotation curve.

Instead of constant projected surface density, many LSBs are found to have instead constant volume density (de Blok 2003). These two views (constant projected surface density, or constant mass volume density) can be consistent if the scale height in the central regions of these LSBs are nearly constant, as for a pancake-type proto galaxy cloud collapse.

(III) Flat rotation curve, as is representative of the outer part of most $\mathrm{Sb} / \mathrm{Sc}$ galaxies.

In this regime, the projected surface density profile should be close to $1 / R$, as shown below. Assume $\Sigma_{\text {proj }}(R)=\frac{\Sigma_{0} R_{0}}{R}$, where $\Sigma_{0}=\Sigma_{\text {proj }}\left(R=R_{0}\right)$, we have $M_{d y n}(R)=\int_{0}^{R} \Sigma_{\text {proj }}(r) 2 \pi r d r=\Sigma_{0} R_{0} 2 \pi R$. Using again $V(R)^{2}=$ $G M_{d y n} / R$, we obtain $V(R)^{2}=G \Sigma_{0} R_{0} 2 \pi R / R$, or $V(R)=\sqrt{G \Sigma_{0} R_{0} 2 \pi}=$ constant. Therefore the flat rotation curve regime corresponds to $1 / \mathrm{R}$ projected surface density distribution.

(IV) The falling rotation curve regime, as representative of the outer part of the early type galaxies.

In this regime the projected surface density $\Sigma_{\text {proj }}(R)$ falls off faster than $1 / \mathrm{R}$, and can be up to $\Sigma_{\text {proj }}=0$ (Keplerian). For the extreme case of Keplerian 
rotation curve due to a concentrated central mass distribution, $\Sigma_{\text {proj }}\left(R_{\text {outer }}\right) \approx$ 0 , and $V(R)^{2}=\frac{G M_{d y n}}{R}$, or $V(R)=\sqrt{\frac{G M_{d y n}}{R}}$, which is indeed falling.

In the secular evolution scenario a typical galaxy starts its life with properties similar to that of an LSB galaxy. The high-z correspondence of the local LSBs appears to be the damped $L_{\alpha}$ system (Wolfe 2001), which also possesses disk-like morphology with large scale length, and a constituting mass component which is gas rich and metal poor.

As the proto galaxy disk contracts and condenses, the global spiral instability develops, which begins the mass-redistribution process that transforms the flat surface density distribution to a more and more centrally concentrated mass distribution. The intermediate stage of this evolution resembles Freeman's type II disks (Freeman 1970), and the later stage with a a 1/R projected surface density resembles a Freeman's type I disk.

As the evolution progresses, the inward-accreted disk stars rise out of the disk plane to become bulge stars. The thermalized three-dimensional bulge mass will have nearly constant volume density and undergoes solid-body rotation. The outer part of the visible disk starts to be drained of matter, producing the falling rotation curve.

This entire evolution sequence therefore corresponds to a galaxy evoling from the lower rotation curve to the upper rotation curve in Figure 1.

The twisted isophotes often found for the Hubble Deep Field galaxies are expected to evolve into the observed variation of major axis with radius for elliptical galaxies (Faber \& Jackson 1976), possibly bypassing the disk formation phase, at least for some galaxies. Like the spiral structure, the twisted isophotes are results of dissipation and differential rotation, and just as in the case of spiral structure, these three-dimensional skewed structures will also lead to a phaseshift between the potential and density distributions, resulting in accelerated secular morphological changes.

\section{Generation and Evolution of the Galaxy Scaling Relations Along the Hubble Sequence}

Galaxy scaling relations (Faber \& Jackson 1976; Tully \& Fisher 1977; Djorgovski \& Davis 1987; Dressler et al. 1987) own their existence first and foremost to the fact that galaxies are equilibrium configurations and satisfy the Virial theorem relation (see, e.g., Binney \& Tremaine 1987). This relation dictates that during any quasi-equilibrium evolution process of a gravitational system, $-E \sim T \sim-V / 2$ where $\mathrm{T}$ is the kinetic energy, $\mathrm{V}$ is the potential energy, and $E=T+V$ is the total energy of the system. The fact that spiral galaxies can remain on the Tully-Fisher relation during secular morphological evolution despite the fact that stars can't radiate about $1 / 2$ of the converted potential energy away, is due to the fact that spiral density wave actually carries 
about $1 / 2$ of the dissipated orbital energy (equal to potential energy) away to the outer disk to be deposited there (Zhang 1999). The existence of a collective dissipation process at the spiral arms also made possible the regulated conversion of orbital velocity to random velocity, so a single kinetic energy (the half that is not being carried away) can enter the Virial relation no matter how it is apportioned between the circular and the random motions.

According to the Virial theorem (we set $\mathrm{G}=1$ in the following derivations of scaling relations) $M_{d y n}=V_{e}^{2} R_{e}$, where $M_{d y n}$ is the dynamical mass of a galaxy, $V_{e}$ is the effective velocity spread which corresponds to the total velocity dispersion for an elliptical galaxy or the maximum rotation velocity for a spiral galaxy, and $R_{e}$ is an effective radius which makes the Virial relation hold.

Define the mean surface brightness of a galaxy $S B$ as $S B \equiv \frac{L}{R_{e}^{2}}$ where $L$ is the luminosity of the galaxy, and the dynamical mass-to-light ratio $M_{d y n} / L$ can be written as $M_{d y n} \equiv\left(M_{d y n} / L\right) * L$. Therefore it follows that

$$
\left(M_{d y n} / L\right)^{2} * L^{2}=M_{d y n}^{2}=V_{e}^{4} R^{2}=V_{e}^{4} \frac{L}{S B},
$$

or

$$
\left(M_{d y n} / L\right)^{2} L=\frac{V_{e}^{4}}{S B} .
$$

Therefore we finally have from

$$
L=V_{e}^{4} \frac{1}{S B} \frac{1}{\left(M_{d y n} / L\right)^{2}} .
$$

We thus see that in order to obtain the classical Tully-Fisher relation, which has $L \propto V_{e}^{4}$, we must have $S B \cdot\left(M_{d y n} / L\right) \sim$ constant. This can be accomplished in two ways: to have $S B$ and $M_{d y n} / L$ each being constant; or, to have the variations of the two factors offset each other during an evolution process.

Traditionally, the former was assumed to be the case (see, e.g. the discussions and references in Shu 1982). It is now known that both these quantities vary considerably for galaxies along the Hubble sequence. The surface brightness is found to be higher for earlier type galaxies (McGaugh \& de Blok 1998 and the references therein), whereas the dynamical mass-to-light is found to be lower for the earlier Hubble types (Zwaan et al. 1995; Bell \& de Jong 2001). The observed opposing trends of variation of surface brightness and dynamical mass-to-light ratio is naturally explained as the outcome of the secular baryonic mass accretion and the increase fraction of luminous baryon mass, especially towards the central region of a galaxy as its Huuble type evolve from late to early. The Tully-Fisher relation can be maintained as long as the increase in surface brightness is accompanied by a corresponding decrease in the dynamical mass to light ratio during the secular evolution process. 
In the secular evolution scenario, since most of the elliptical galaxies (i.e. those lower luminosity, so-called disky ellipticals) are the end results of evolution from the initial condition of disks, we speculate that spiral galaxies may satisfy similar kind of fundamental plane relation just as ellipticals. This has been found to be so (see, e.g., Pharasyn, Simien, \& Heraudeau 1997). The fundamental plane relation for spirals can likewise be derived from the Virial theorem. Starting from

$$
V_{e}^{2}=G \frac{M_{d y n}}{R_{e}}
$$

we have

$$
V_{e}^{2}=G \frac{M_{d y n}}{L} \frac{L}{R_{e}}=L^{1 / 2}\left(\frac{L}{R_{e}^{2}}\right)^{1 / 2} \frac{M_{d y n}}{L}
$$

which leads to

$$
10 \log V_{e}=-(1+2 \beta) M_{t}-\mu_{e}+\text { constant, }
$$

where $M_{t}$ is the absolute magnitude and $\mu_{e}$ is the face-on average surface brightness in mag/arcsec ${ }^{2}$, and where we have assumed $\mathrm{M}_{d y n} / \mathrm{L} \propto L^{\beta}$. Pharasyn et al. (1997) found that fitting I band and K band data of a sample of spiral galaxies to this relation resulted in $\beta \approx-0.15$, i.e. the mass-to-light ratio slightly decreases with increasing luminosity, which is consistent with what we would expect from a secular evolution picture, i.e. as the secular evolution advances, $\mathrm{L}$ increases and $\mathrm{M}_{d y n} / \mathrm{L}$ decreases.

The fundamental plane relation for elliptical galaxies and bulges were first obtained by Faber et al. (1987), Djorgovski \& Davis (1987), and Dressler et al. (1987). One type of the fundamental plane relation for ellipticals can be written as (c.f. equation 2a and 2b of Djorgovski \& Davis 1987)

$$
L \sim V_{e}^{3.45} S B^{-0.86} .
$$

This relation can be re-written into the form

$$
M_{t}\left(R_{e}\right)=-8.62\left(\log V_{e}+0.1 \mu_{e}\right)+\text { constant }
$$

which can be further written as we arrive at

$$
10 \log V_{e}=-1.25 M\left(R_{e}\right)-\mu_{e}+\text { constant. }
$$

Comparing equations (10) and (13), we see that apart from a possible difference in the "constant" term (accounting for the change in total luminosity), the only difference between the spiral and elliptical fundamental plane relations is in the different sign of the mass-to-light ratio exponent $\beta$ : $\beta=-0.15$ for spirals and $\beta=0.13$ for ellipticals and bulges, where $\beta$ is defined through 
$M_{d y n} / L=L^{\beta}$. This difference is apparently brought about by the fact that spiral galaxies still have varying reserves of baryonic dark matter to form stars, therefore as the secular evolution proceeds (and therefore L increases) the mass-to-light ratio decrease; whereas elliptical galaxies have essentially exhausted their central baryonic dark matter supply, thus the ellipticals in more advanced stage of evolution (which also generally have larger L) will experience greater degree of dimming, which is reflected in the increase of mass-tolight ratio with $\mathrm{L}$.

This research was supported in part by funding from the Office of Naval Research.

\section{References}

Andredakis, Y.C., Peletier, R.F., \& Balcells, M. 1995, MNRAS, 275, 874

Binney, J., \& Tremaine, S. 1987, Galactic Dynamics (Princeton:Princeton Univ. Press)

Bell, E.F. \& de Jong, R.S. 2001, ApJ, 550, 212.

Combes, F., \& Sanders, R.H. 1981, A\&A, 96, 164

Conselice, C.J., Kershady, M.A., Dickinson, M., \& Papovich, C. 2003, AJ, 126, 1183

de Blok, W.J.G. 2003, presented in Dark Matter in Galaxies, IAUS 220

Djorgovski, S. \& Davis, M. 1987, ApJ, 313, 59

Dressler, A. et al. 1987, ApJ, 313, 42

Faber, S.M., et al. in Nearly Normal Galaxies, ed. S.M. Faber (New York: Springer), 175

Faber, S.M., \& Jackson, R.E. 1976, ApJ, 204, 668

Freeman, K.C. 1970, ApJ, 160, 811

Hamabe, M., \& Kormendy, J. 1987, in Structure and Dynamics of Elliptical Galaxies, ed T. de Zeeuw (IAU), 379

Jablonka, P., Gorgas, J., \& Goudfrooij, P. 2002, Ap\&SS, 281, 367

Kormendy, J. 1982, in 12th Advanced Course of the SSAA, eds. L. Martinet, \& M. Mayor (Geneva Observatory: Geneva), 115

Kormendy, J., \& Kennicutt, R. 2004, ARA\&A, in press

Lynden-Bell, D., \& Kalnajs, A.J., 1972, MNRAS, 157, 1

McGaugh, S.S., \& de Blok, W.J.G. 1998, ApJ, 499, 41

Peebles, J. 1983, Physical Cosmology (Princeton: Princeton Univ. Press)

Persic, M., Salucci, P., \& Stel, F. 1996, MNRAS, 281, 27

Pharasyn, A., Simien, F., \& Heraudeau, Ph. 1997, in Dark and Visible Matter in Galaxies, ASP Conf. Series 117, eds M. Persic \& P Salucci (San Francisco: ASP), 180

Shu, F.S., 1982, The Physical Universe: An Introduction to Astronomy (Mill Valley: University Science Books)

Toomre, A., \& Toomre, J. 1972, ApJ, 178, 623

Tully, R.B., \& Fisher, J.R. 1977, A\&A, 54, 661

Wielen, R. 1977, A\&A, 60, 263

Wolfe, A.M., 2001, in Galaxy Disks and Disk Galaxies, eds J.G. Funes, S.J. \& E.M. Corsini (San Francisco: ASP), 619

Zhang, X. 1996, ApJ, 457, 125; 1998, ApJ, 499, 93; 1999, ApJ, 518, 613

Zhang, X., 2002, Ap\&SS, 281, 281

Zhang, X. 2003, JKAS, 36, 223

Zhang, X., Lee, Y., Bolatto, A., \& Stark, A.A., 2001, ApJ, 553, 274

Zwaan, M.A., van der Hulst, J.M., de Bolk, W.J.G., \& McGaugh, S.S. 1995, MNRAS, 273, L35 